\title{
Cellular Networks as Models for Planck-Scale Physics
}

\author{
Manfred Requardt \\ Institut für Theoretische Physik \\ Universität Göttingen \\ Bunsenstrasse 9 \\ 37073 Göttingen Germany
}

\begin{abstract}
Starting from the working hypothesis that both physics and the corresponding mathematics have to be described by means of discrete concepts on the Planck scale, one of the many problems one has to face in this enterprise is to find the discrete protoforms of the building blocks of our ordinary continuum physics and mathematics. We base our own approach on what we call 'cellular networks', consisting of cells (nodes) interacting with each other via bonds (figuring as elementary interactions) according to a certain 'local law'. Geometrically our dynamical networks are living on graphs. Hence a substantial amount of the investigation is devoted to the developement of various versions of discrete (functional) analysis and geometry on such (almost random) webs. Another important topic we address is a suitable concept of intrinsic (fractal) dimension on erratic structures of this kind. In the course of the investigation we make comments concerning both different and related approaches to quantum gravity as, say, the spin network framework. It may perhaps be said that certain parts of our programme seem to be a realisation of ideas sketched by Smolin some time ago (see the introduction).
\end{abstract}




\section{Introduction}

There exists a certain suspicion in parts of the scientific community that nature may be discrete or rather "behaves discretely" on the Planck scale. But even if one is willing to agree with this "working philosophy", it is far from being evident what this vague metaphor actually has to mean or how it should be implemented into a concrete and systematic inquiry concerning physics and mathematics in the Planck regime.

There are basically two overall attitudes as to "discreteness on the Planck scale", the one comprising approaches which start (to a greater or lesser degree) from continuum concepts (or more specifically: concepts being more or less openly inspired by them) and then try to detect or create modes of "discrete behavior" on very fine scales, typically by imposing quantum theory in full or in parts upon the model system or framework under discussion.

There are prominent and very promising candidates in this class like e.g. 'string theory' or 'loop quantum gravity'. Somewhat intermediate is a more recent version (or rather: aspect) of the latter approach, its 'polymer' respectively 'spin network' variants. As these approaches are meanwhile widely known we refrain from citing from the vast corresponding literature. We recommend instead as more recent reviews as to the latter approach, containing some cursory remarks about the former together with a host of references, [1] and [2] and, as a beautiful introduction to the conceptual problems of quntum gravity in general, [3].

On the other hand one could adopt an even more speculative and radical attitude and approach the Planck regime from more or less the opposite direction by developing a framework almost from scratch which has "discreteness" already built in into its very building blocks and then try to reconstruct, working, so to speak, "bottom up", all the continuum concepts of ordinary space-time physics as sort of "collective quantities' like e.g. 'collective excitations' via the cooperation of many microscopic (discrete) degrees of freedom. If one is very bold one could even entertain the idea that the quantum phenomena as such are perhaps not the eternal and irreducible principles as they are still viewed today by the majority of physicists but, to the contrary, may emerge as derived and secondary concepts, together with gravitation, from a more primordial truly discrete and 'combinatorial' theory. As to corresponding strategies see also the perhaps prophetical remarks of R.Penrose in [4 or the bundle of ideas presented in [5] beginning roughly at page 267. To the wider context belong also the ideas of Sorkin, Balachandran et al. (see e.g. [6]), while the approach of 't Hooft ([7]) is noteworthy because of its emphasis on the possible usefulness of cellular automata (i.e. simpler and more rigid variants of our cellular networks).

We would like to add a perhaps clarifying remark concerning reference [5]. The mentioned ideas are sketched almost at the end of a long contribution and we stumbled over them only very recently (February 1998). One could perhaps venture to say that the framework we are going to develop in the following and in related papers (see [8]) 
to [11] is, at least in part, an implementation of Smolin's programme. This concerns in particular one of the main motivations of both Smolin's and our approach, namely the hope that, proceeding in this way, the peculiar form of nonlocality or entanglement observed in quantum theory can actually be implemented and thus better understood.

It goes without saying that such a radical approach is beyond the reach of direct experimental verification in the strict sense for the foreseeable future (as is the case with the other frameworks mentioned above). As a substitute one rather has to rely on inner theoretical criteria, among other things, the capability to generate the hierarchy of increasingly complex patterns we are observing in nature or in our present day 'effective theories', which describe the various regimes many orders of magnitude away from the Planck scale, while introducing, on the other hand, as few simple and elementary assumptions as possible. More specifically, one would like such a framework to provide clues how the continuum concepts of ordinary space-time physics/mathematics may emerge in a natural manner from their respective 'discrete protoforms'.

Another more aesthetic criterion would be a kind of natural convergence of the different approaches towards a common substructure which is discrete in a really primordial way. Indications for such a convergence can, in our view, be detected in the various lines of research going on presently. 'spin networks' or 'polymer states' are e.g. cases in point where modes of discreteness do emerge from a at first glance continuous environment. Furthermore it may well be that 'string field theory' will turn out to live on a more discrete and singular substratum than presently suspected (some speculative remarks pointing in this direction can e.g. be found at the end of [12]), a catchword being 'fractal geometry'. A brief but illuminating analysis concerning such a possible convergence in the future towards a decidedly discrete and 'combinatorial' common limit has been given in section 8 of [1]. A bundle of related ideas with which we sympathize is developed by Nottale (see e.g. [13]).

In the following sections we will embark on the developement of a conceptual framework which has a pronouncedly combinatorial flavor and makes contact with various branches of modern discrete mathematics as e.g. algebraic combinatorics, (random)graph theory or noncommutative geometry. The paper is more or less divided into three parts. The first part deals with the dynamics of 'cellular networks' we will introduce below. Insofar our approach is different from perhaps more canonical approaches which rely in a more or less open way on ideas being already employed in the continuum versions as e.g. 'fields', 'path integrals' or a relative regular substratum like a periodic lattice structure. Quite to the contrary, we regard most of these concepts as 'emergent structures' according to our working philosophy. In other words, these patterns need not have identifiable counterparts on the primordial scale.

As to such questions of principle we share the philosophy of e.g. Smolin, Ashtekar (and quite a few others, see e.g. section 6.1 in [14]) that if one agrees that a continuum theory has to be understood as the coarse grained limit of an underlying 'critical' discrete and more fundamental theory, there is no sufficient reason to surmise that 
the primordial building blocks or the dynamics at perhaps the ultimate bottom of our universe will share a lot of similarities with the (almost) continuum concepts of our 'effective theories' living in a regime of at most intermediate energies many orders of magnitude away from the Planck regime.

The second part of our paper consists of a presentation of a couple of ways how one can introduce versions of discrete analysis and geometry in such erratic environments we are going to study in the following. At the same time we undertake to compare our approach with other existing ones (being, in part, derivatives of noncommutative geometry in the sense of Connes et al).

In the last part we develop dimensional concepts on such discrete spaces. We would like to stress the point that we consider it to be of crucial importance not to base such concepts on anything which could be identified as a variant of a continuum or embedding space. As to these ideas much more can be found in [8], in particular concerning various conceptual relations to fractal geometry.

We want to close this introduction with a perhaps clarifying remark. To discretize continuum quantum gravity (or rather certain models), e.g. to put it on a lattice or triangulate it, is of course not new, but most of the approaches we are aware of discretize continuum theories living in a fixed embedding space with a definite dimension in the standard sense. Furthermore the kind of discretizations being employed are typically relatively regular while in our approach the underlying graphs are rather random structures with vertices and bonds as dynamical variables which can even be switched on or off. As a consequence the dimension of our networks is an 'emergent' and dynamical, perhaps even fractal, property of the system. To mention only two representative paper of the many others employing or reviewing strategies of the former type see e.g. [15] or [16] and for more references the thesis of Bakker ([17]), the lecture of T.D.Lee ([18) should perhaps also be mentioned in this context. This does not of course mean that there are no relations between these different approaches but we prefer to discuss them elsewhere in order to keep the paper readable.

\section{The Cellular Network Environment}

In this section we will sketch the type of model systems on which the following analysis will be based. As already said in the introduction we will start from a rather primordial level, trying to make no allusions whatsoever to continuum concepts. We will then show how protoforms of ideas and notions, playing a key role in ordinary continuum physics/mathematics emerge in a relatively natural and unforced way from this framework. Cases in point are e.g. concepts like 'dimension', 'differential structure', the idea of 'physical points' (being endowed with an internal structure), the web of which establishes the substratum of macroscopic space-time, and other geometrical/topological notions. The framework turns out to be even rich enough to support a full fledged kind of 'discrete functional analysis', comprising e.g. 'Laplace-, Dirac operators' and all that. It is perhaps particularly noteworthy that an advanced 
structure like 'Connes' spectral triple' shows up in a very natural way within this context.

Besides the reconstruction of basic concepts of continuum physics and mathematics another goal is to describe the micro dynamics going on in this discrete substratum over (in) which macroscopic space-time is floating as a kind of coarse grained 'superstructure', the formation of 'physical points' and their mutual entanglement, yielding the kind of 'near-/far-order' or 'causal structure' we are used to from continuum space-time.

To this end we view this substratum as, what we like to call, a 'cellular network', consisting of 'nodes' and 'bonds'. The nodes are assumed to represent certain elementary modules (cells or "monads") having a discrete, usually simple, internal state structure, the bonds modeling elementary direct interactions among the nodes. As an important ingredient, these bonds are dynamical insofar as they are capable to be in a (typically limited) number of 'bond states', thus implementing the varying strength of the mutual interactions among the cells.

It is a further characteristic of our model class that these interactions are not only allowed to vary in strength but, a fortiori, can be switched off or on, depending on the state of their local environment. In other words, stated in physical terms, bonds can be created or annihilated in the course of network evolution, which (hopefully) enables the system to undergo 'geometric phase transitions' being accompanied by an 'unfolding' and 'pattern formation', starting e.g. from a less structured chaotic initial phase. To put it briefly: in contrast to, say, 'cellular automata', which are relatively rigid and regular in their wiring and geometric structure (in particular: with the bonds typically being non-dynamical), our cellular networks do not carry such a rigid overall order as an external constraint (e.g. a regular lattice structure); their "wiring" is dynamical and thus behaves randomly to some extent. The clue is that order and modes of regularity are hoped to emerge via a process of 'self-organization'.

\subsection{Definition (Class of Cellular Networks)}

1. "Geometrically" our networks represent at each fixed 'clock time' a 'labeled graph', i.e. they consist of nodes $\left\{n_{i}\right\}$ and bonds $\left\{b_{i k}\right\}$ or $\left\{d_{i k}\right\}$ (see the next section), with the bond $b_{i k}$ connecting the nodes (cells) $n_{i}, n_{k}$. We assume that the graph has neither elementary loops nor multi-bonds, that is, only nodes with $i \neq k$ are connected by at most one bond.

2. At each site $n_{i}$ we have a local node state $s_{i} \in q \cdot \mathbb{Z}$ with $q$, for the time being, a certain not further specified elementary quantum. The bond variables $J_{i k}$, attached to $b_{i k}$, are in the most simplest cases assumed to be two- or threevalued, i.e. $J_{i k} \in\{ \pm 1\} \quad$ or $\quad J_{i k} \in\{ \pm 1,0\}$

Remarks 
1. In the proper graph context the notions 'vertex' and 'edge' are perhaps more common (see e.g. [19]). As to some further concepts used in graph theory see below.

2. This is, in some sense, the simplest choice one can make. It is an easy matter to employ instead more complicated internal state spaces like, say, groups, manifolds etc. One could in particular replace $\mathbb{Z}$ by one of its subgroups or impose suitable boundary conditions.

3. In the following section we will give the bonds $b_{i k}$ an 'orientation', i.e. (understood in an precise algebraic/geometric sense) $b_{i k}=-b_{k i}$. This implies the compatibility conditions $J_{i k}=-J_{k i}$.

In a next step we have to impose a dynamical law on our model network. In doing this we are of course inspired by 'cellular automaton laws' (see e.g. 20]). The main difference is however that in our context also the bonds are dynamical degrees of freedom and that, a fortiori, they can become dead or alive (active or inactive), so that the whole net is capable of performing drastic topological/geometrical changes in the course of clock time.

A particular type of a dynamical 'local law' is now introduced as follows: We assume that all the nodes/bonds at '(clock) time' $t+\tau, \tau$ an elementary clock time step, are updated according to a certain local rule which relates for each given node $n_{i}$ and bond $b_{i k}$ their respective states at time $t+\tau$ with the states of the nodes/bonds of a certain fixed local neighborhood at time $t$.

It is important that, generically, such a law does not lead to a reversible time evolution, i.e. there will typically exist attractors in total phase space (the overall configuration space of the node and bond states).

A crucial ingredient of our network laws is what we would like to call a 'hysteresis interval'. We will assume that our network, called in the following $Q X$ ("quantum space)", starts from a densely entangled 'initial phase' $Q X_{0}$, in which practically every pair of nodes is on average connected by an 'active' bond, i.e. $J_{i k}= \pm 1$. Our dynamical law will have a built-in mechanism which switches bonds off (more properly: sets $J_{i k}=0$ ) if local fluctuations among the node states become too large. Then there is hope that this mechanism may trigger an 'unfolding phase transition', starting from a local seed of spontaneous large fluctuations towards a new phase (an attractor) carrying a certain 'super structure', which we would like to relate to the hidden discrete substratum of space-time (points).

One example of such a law is given in the following definition.

2.2 Definition (Local Law) At each clock time step a certain 'quantum' $q$ is transported between, say, the nodes $n_{i}, n_{k}$ such that

$$
s_{i}(t+\tau)-s_{i}(t)=q \cdot \sum_{k} J_{k i}(t)
$$


(i.e. if $J_{k i}=+1$ a quantum $q$ flows from $n_{k}$ to $n_{i}$ etc.)

The second part of the law describes the back reaction on the bonds (and is, typically, more subtle). This is the place where the so-called 'hysteresis interval' enters the stage. We assume the existence of two 'critical parameters' $0 \leq \lambda_{1} \leq \lambda_{2}$ with:

$$
\begin{gathered}
J_{i k}(t+\tau)=0 \quad \text { if } \quad\left|s_{i}(t)-s_{k}(t)\right|=:\left|s_{i k}(t)\right|>\lambda_{2} \\
J_{i k}(t+\tau)= \pm 1 \quad \text { if } \quad 0< \pm s_{i k}(t)<\lambda_{1}
\end{gathered}
$$

with the special proviso that

$$
J_{i k}(t+\tau)=J_{i k}(t) \quad \text { if } \quad s_{i k}(t)=0
$$

On the other side

$$
J_{i k}(t+\tau)=\left\{\begin{array}{ll} 
\pm 1 & J_{i k}(t) \neq 0 \\
0 & J_{i k}(t)=0
\end{array} \quad \text { if } \quad \lambda_{1} \leq \pm s_{i k}(t) \leq \lambda_{2}\right.
$$

In other words, bonds are switched off if local spatial charge fluctuations are too large, switched on again if they are too small, their orientation following the sign of local charge differences, or remain inactive.

\section{Remark}

1. The reason why we do not choose the "current" $q \cdot J_{i k}$ proportional to the "voltage difference" $\left(s_{i}-s_{k}\right)$ as e.g. in Ohm's law is that we favor a non-linear network which is capable of self-excitation and self-organization rather than self-regulation around a relatively uninteresting equilibrium state! The balance between dissipation and amplification of spontaneous fluctuations has however to be carefully chosen ("complexity at the edge of chaos")

2. We presently have emulated these local network laws on a computer. It is not yet clear whether this simple network law does already everything we are expecting. In any case, it is fascinating to observe the enormous capability of such intelligent networks to find attractors very rapidly, given the enormous accessible phase space

3. In the above class of laws a direct bond-bond-interaction is not yet implemented. We are prepared to incorporate such a contribution in a next step if it turns out to be necessary. In any case there are not so many ways to do this in a sensible way. Stated differently, the class of possible physically sensible interactions is perhaps not so numerous.

4. Note that - in contrast to e.g. Euclidean lattice field theory - the so-called 'clock time' $t$ is, for the time being, not standing on the same footing as potential "coordinates" in the network (e.g. curves of nodes/bonds). We rather suppose 
that so-called 'physical time' will emerge as sort of a secondary collective variable in the network, i.e. being different from the clock time (while being of course functionally related to it).

In our view Remark 4 is consistent with the spirit of relativity. What Einstein was really teaching us is that there is a (dynamical) interdependence between what we experience as space respectively time, not that they are absolutely identical! In any case, the assumption of an overall clock time is at the moment only made just for convenience in order to make the model system not too complicated. If our understanding of the complex behavior of the network dynamics increases, this assumption may be weakened in favor of a possibly local and/or dynamical clock frequency. A similar attitude should be adopted concerning concepts like 'Lorentz-(In)Covariance' which we also consider as 'emergent' properties. It is needless to say that it is of tantamount importance to understand the way how these patterns do emerge from the relatively chaotic background, questions which will be addressed in future work.

As can be seen from the definition of the cellular network, a full scale investigation of its behavior separates quite naturally into two parts of both a different mathematical and physical nature. The first one comprises its more geometric/algebraic content in form of large static graphs and their intricate structure (at, say, arbitrary but fixed clock time), thus neglecting the details of the internal states of bonds and nodes, the other one conveys a more dynamical flavor, i.e. analyzing and keeping track of the topological/geometrical change and pattern formation in the course of clock time. Both parts represent an intricately entangled bundle of complicated problems and do require the developement or application of a fair amount of quite advanced (discrete) mathematics. Therefore we prefer to concentrate in the following on the former part; as to the latter part a certain impression of the impending problems is given in [11] which, however, should be considered only as a preliminary draft.

Before we embark on studying in more detail the 'pregeometric' patterns in cellular networks we want to briefly comment on the more general structure of evolution laws in such systems. The above one is only one candidate from a whole class of such laws. For one, it is quite evident that the 'local state spaces' living over the respective nodes and bonds can be chosen in a more general way. For another, the local dynamical law can be chosen more general.

\subsection{Definition (General Local Law on Cellular Networks):}

Each node $n_{i}$ can be in a number of internal states $s_{i} \in \mathcal{S}$. Each bond $b_{i k}$ carries a corresponding bond state $J_{i k} \in \mathcal{J}$. Then the following general transition law is assumed to hold:

$$
\begin{gathered}
s_{i}(t+\tau)=l l_{s}\left(\left\{s_{k}(t)\right\},\left\{J_{k l}(t)\right\}\right) \\
J_{i k}(t+\tau)=l l_{J}\left(\left\{s_{l}(t)\right\},\left\{J_{l m}(t)\right\}\right) \\
(\underline{S}, \underline{J})(t+\tau)=L L((\underline{S}, \underline{J})(t))
\end{gathered}
$$


where $l l_{s}, l l_{J}$ are two maps (being the same over the whole graph) from the state space of a local neighborhood of the node or bond on the lhs to $\mathcal{S}, \mathcal{J}$, yielding the updated values of $s_{i}$ and $J_{i k} . \underline{S}$ and $\underline{J}$ denote the global states of the nodes and bonds and $L L$ the global law built from the local laws at each node or bond.

Irrespectively of the technical details of the dynamical evolution law under discussion it should emulate the following, in our view crucial, principles, in order to match certain fundamental requirements concerning the capability of 'emergent' and 'complex' behavior.

1. As is the case with, say, gauge theory or general relativity, our evolution law on the primordial level should encode the mutual interaction of two fundamental substructures, put sloppily: "geometry" acting on "matter" and vice versa, where in our context "geometry" is assumed to correspond in a loose sense with the local and/or global bond states and "matter" with the structure of the node states. (We will not comment further on this working philosophy at the moment as it represents the basis of forthcoming work).

2. By the same token the above selfreferential dynamical circuit of mutual interactions is expected to favor a kind of 'undulating behavior' or 'selfexcitation' above a return to some uninteresting 'equilibrium state' which is frequently found among systems consisting of a single component which directly acts back on itself. This propensity for the 'autonomous' generation of undulation patterns is in our view an essential prerequisite for some form of "protoquantum behavior" we hope to recover on some coarse grained and less primordial level of the network dynamics.

3. In the same sense we expect the possibility of switching on and off of bonds to generate a kind of "protogravity".

We close this section with a short aside concerning the definition of evolution laws of 'spin networks' by Markopoulou, Smolin and Borissov (see [21] or [22]). As in our case there are more or less two possibilities: treating evolution laws within an integrated space-time formalism or regard the network as representing space alone with the time evolution being implanted via some extra principle ( which is the way we have chosen above). Closely connected with this question is the developement of a suitable concept of dimension in this context. We start to develop our personal concept in the last section of this paper. More information can be found in [8]. As the interrelation of these various approaches and frameworks is both very interesting and presently far from obvious we plan to compare them elsewhere. 


\section{Discrete Analysis on Graphs and Networks}

In the following we will show that despite of its discreteness graphs and networks are capable of supporting a surprisingly rich differential and geometric structure, so that the catchword 'discrete analysis' in the headline of this section is no exaggeration. One can even develop a full fledged 'discrete functional analysis' with Hilbert spaces, graph Laplacian, Dirac operator etc. This then leads into the fascinating field of describing geometric structures and patterns on the graph with the help of functional analytic tools (see [10]).

While our original approach has been developed from a somewhat different perspective there emerged nevertheless in the course of evolution of our framework various regions of contact and overlap with other approaches like e.g. what is called 'noncommutative geometry' (see e.g. [23] or [24]; as a brief but concise introduction we recommend also [25]). If a certain part of this highly abstract machinery is applied to, say, discrete sets, one gets a version of discrete calculus which is still a relatively abstract scheme as long as it is not interpreted within a concrete model theory. Such an abstract calculus has been developed by e.g. Dimakis, Mueller-Hoissen et al. (see for example [26]). In the following we will attempt, among other things, to relate these in some respects different, in other respects related approaches with our own framework.

We will develop below various schemes with only the first one being related in some respects to noncommutative geometry. The other ones are standing on a more or less independent footing. In this first approach context we treat the network as a static labelled graph, consisting solely of nodes and bonds. We start with some graph theoretical concepts:

\subsection{Definition (Simple Locally Finite (Un)directed Graph):}

1. We write the 'simple' graph as $G:=(V, E)$ where $V$ is the countable set of nodes $\left\{n_{i}\right\}$ (or vertices) and $E$ the set of bonds (edges). The graph is called simple if there do not exist elementary 'loops' and 'multiple edges', in other words: each existing bond connects two different nodes and there exists at most one bond between two nodes. (We could of course also discuss more general graphs). Furthermore, for simplicity, we assume the graph to be connected, i.e. two arbitrary nodes can be connected by a sequence of consecutive bonds called an 'edge sequence' or 'walk'. A minimal edge sequence, that is one with each intermediate node occurring only once, is called a 'path' (note that these definitions may change from author to author).

2. We assume the graph to be 'locally finite', that is, each node is incident with only a finite number of bonds. Sometimes it is useful to make the stronger assumption that this 'vertex degree', $v_{i}$, (number of bonds being incident with 
$\left.n_{i}\right)$, is globally bounded away from $\infty$.

3. One can give the edges both an 'orientation' and a 'direction' (these two slightly different geometric concepts are frequently intermixed in the literature). In our context we adopt the following convention: If two nodes $n_{i}, n_{k}$ are connected by a bond, we interpret this as follows: There exists a 'directed bond', $d_{i k}$, pointing from $n_{i}$ to $n_{k}$ and a directed bond, $d_{k i}$, pointing in the opposite direction. In an algebraic sense, which will become clear below, we call their 'superposition'

$$
b_{i k}:=d_{i k}-d_{k i}=-b_{k i}
$$

the corresponding 'oriented bond' (for obvious reasons; the directions are fixed while the orientation can change its sign). In a sense the above reflects the equivalence of an 'undirected graph' with a 'directed multi-graph' having two directed bonds pointing in opposite directions for each undirected bond.

We now take the elementary building blocks $\left\{n_{i}\right\}$ and $\left\{d_{i k}\right\}$ as basis elements of a certain hierarchy of vector spaces over, say, $\mathbb{C}$ with scalar product

$$
<n_{i}\left|n_{k}>=\delta_{i k} \quad<d_{i k}\right| d_{l m}>=\delta_{i l} \cdot \delta_{k m}
$$

3.2 Definition (Vertex-, Edge-Space): The vector spaces (or modules) $C_{0}$ and $C_{1}$, consist of the finite sums

$$
f:=\sum f_{i} n_{i} \quad \text { and } \quad g:=\sum g_{i k} d_{i k}
$$

$f_{i}, g_{i k}$ ranging over a certain given field like e.g. $\mathbb{C}$ or ring like e.g. $\mathbb{Z}$ in case of a module.

Remark:

1. These spaces can be easily completed to Hilbert spaces (as it was done in [10]) by assuming

$$
\sum\left|f_{i}\right|^{2}<\infty \quad \sum\left|g_{i k}\right|^{2}<\infty
$$

if one chooses the field $\mathbb{C}$.

2. One can continue this row of vector spaces in a way which is common practice in, say, algebraic topology (for more details see below). In this context they could equally well be called 'chain complexes'.

3. Evidently the above vector spaces could as well be viewed as discrete function spaces over the node-, bond set with $n_{i}, d_{i k}$ representing the elementary indicator functions. 
In the same spirit we can now introduce two linear maps between $C_{0}, C_{1}$ called for obvious reasons 'boundary-' and 'coboundary map'. On the basis elements they act as follows:

\subsection{Definition/Observation ((Co)boundary Operator):}

$$
\begin{aligned}
\delta: d_{i k} \rightarrow n_{k} \quad \text { hence } \quad b_{i k} & \rightarrow n_{k}-n_{i} \\
d: n_{i} & \rightarrow \sum_{k}\left(d_{k i}-d_{i k}\right)=\sum_{k} b_{k i}
\end{aligned}
$$

and linearly extended. That is, $\delta$ maps the directed bonds $d_{i k}$ onto the terminal node and $b_{i k}$ onto its 'boundary', while $d$ maps the node $n_{i}$ onto the sum of the 'ingoing' directed bonds minus the sum of the 'outgoing' directed bonds or on th sum of 'oriented' ingoing bonds $b_{k i}$.

The following results show, that these definitions lead in fact to a kind of 'discrete differential calculus'.

3.4 Observation (Discrete Differential Forms): From the above it follows that

$$
d f=d\left(\sum f_{i} n_{i}\right)=\sum_{k, i}\left(f_{k}-f_{i}\right) d_{i k}
$$

One could now enter the field of true discrete functional analysis by defining the so-called 'graph Laplacian'.

\subsection{Definition/Observation (Graph Laplacian):}

$$
\delta d f=-\sum_{i}\left(\sum_{k} f_{k}-v_{i} \cdot f_{i}\right) n_{i}=-\sum_{i}\left(\sum_{k}\left(f_{k}-f_{i}\right)\right) n_{i}=:-\Delta f
$$

where $v_{i}$ denotes the node degree or 'valency' defined above and the $k$-sum extends over the nodes adjacent to $n_{i}$.

As to more results along these lines of reasoning see [10]. In the following we would however prefer to develop a framework which is more in the spirit of 'discrete geometry' or abstract 'combinatorial topology'.

\subsection{The Graded Semi-Differential Algebra of Strings or Walks}

The elementary algebraic/geometric building blocks of our framework will be the nodes and (un)directed bonds. In a next step we use them to form 'edge sequences (walks)' or 'strings'. 
3.6 Definition/Observation (Admissible Strings): With $C_{0}, C_{1}$ denoting the vector spaces spanned by the nodes and bonds, $C_{k}$ comprises finite sums of admissible edge sequences consisting of $k$ consecutive edges or $(k+1)$ consecutive nodes. We write them as

$$
d_{i_{0} i_{1}} \cdot d_{i_{1} i_{2}} \cdots d_{i_{k-1} i_{k}} \quad \text { or } \quad n_{i_{0}} \ldots n_{i_{k}}
$$

where by admissible we mean that each pair of consecutive nodes is connected by a bond. The multiplication sign standing between the respective edges is explained below and the above strings are now interpreted as normalized basis elements in the vector space $C_{k}$ over, say, $\mathbb{C}$.

Remark: Note that the repeated occurrence of a particular node or bond in such a string is not forbidden, whereas two consecutive nodes are always different. A fortiori a substring of the form $d_{i k} \cdot d_{k i}$ or $n_{i} n_{k} n_{i}$ is admissible. Such strings or substrings are algebraically useful if one wants to have the notion of 'inverse string' and a corresponding multiplication structure (thinking of more general algebraic concepts like e.g. groupoids etc.).

We can now extend the (co)boundary maps $\delta, d$ to consecutive pairs of spaces, $C_{k}, C_{k+1}$ by combining geometric imagery with experience from algebraic topology.

3.7 Definition/Observation: With a certain admissible string $n_{0} \ldots n_{k}$ given (for notational simplicity we write $k$ instead of $i_{k}$ ) we define $\delta, d$ as follows:

$$
\delta\left(n_{o} \ldots n_{k}\right):=\sum_{i=0}^{k}(-1)^{i} n_{0} \ldots \widehat{n_{i}} \ldots n_{k}
$$

where the hat means that the respective node has to be deleted with the proviso that the string is mapped to zero if $n_{i-1}, n_{i+1}$ are not connected by a bond, i.e. if the new string is not admissible.

The extension of the coboundary operator runs as follows:

$$
d\left(n_{0} \ldots n_{k}\right):=\sum_{n_{\nu}, i}(-1)^{i} n_{0} \ldots n_{i-1} n_{\nu} n_{i} \ldots n_{k}
$$

where $i$ runs from 0 to $(k+1)$ and admissible nodes are inserted before the node $n_{i}$ or for $i=(k+1)$ after the last node $n_{k}$. The insertion runs over all nodes being connected with both $n_{i-1}$ and $n_{i}$.

The geometric picture behind this scheme is quite transparent. A given string in $C_{k}$ is mapped onto a superposition of strings in $C_{k-1}$ or $C_{k+1}$ with appropriate weights as prefactors.

After these preparatory steps we can now set up a certain discrete differential structure with a strong geometric flavor. We have the $\mathbb{N}_{0}$-graded vector space

$$
C:=\sum C_{k} \quad k \in \mathbb{N} \cup\{0\}
$$


with the 'raising' and 'lowering' operators $d$ and $\delta$, mapping the 'level sets' of homogeneous elements, $C_{k}$, into each other.

In a first step we make $C$ into a 'graded algebra':

3.8 Definition/Observation (Graded Algebra): We define multiplication of homogeneous elements via 'concatenation', i.e:

$$
\left(n_{i_{0}} \ldots n_{i_{k}}\right) \cdot\left(n_{j_{0}} \ldots n_{j_{l}}\right)=\left(n_{i_{0}} \ldots\left(n_{i_{k}} \cdot n_{j_{0}}\right) \ldots n_{j_{l}}\right) \in C_{k+l}
$$

where $n_{i_{k}} \cdot n_{j_{0}}$ means the natural algebra product structure on $C_{0}$, i.e. pointwise multiplication

$$
f \cdot g=\left(\sum f_{i} n_{i}\right) \cdot\left(\sum g_{i} n_{i}\right)=\sum f_{i} g_{i} n_{i}
$$

that is $n_{i_{k}} \cdot n_{j_{0}}=\delta_{i_{k} j_{0}} n_{j_{0}}$.

Geometrically this describes the glueing together of strings or walks, the product being zero if the end node of the first string is different from the initial node of the second string (in other words, this algebra has a lot of 'zero divisors').

As a consequence $C$ has a natural 'bimodule structure' over $C_{0}$.

\subsection{Corollary (Bimodule Structure): With}

$$
\begin{gathered}
n_{i} \cdot\left(n_{i} \ldots n_{k}\right)=\left(n_{i} \cdot n_{1}\right) n_{2} \ldots n_{k}=\delta_{i 1} \cdot n_{1} \ldots n_{k} \\
\left(n_{1} \ldots n_{k}\right) \cdot n_{i}=n_{1} \ldots\left(n_{k} \cdot n_{i}\right)=\delta_{k i} \cdot\left(n_{1} \ldots n_{k}\right)
\end{gathered}
$$

and linear extension $C$ becomes a bimodule over the algebra $C_{0}$. Algebraically $n_{1}, n_{k}$ play the role of 'left-' and 'right-'identities with respect to the above string.

In order to exhibit the connection to differential calculus in the style of Connes or Dimakis et al., we make the following observation concerning the interplay of algebraic and differential structure.

3.10 Observation: Given an admissible string $n_{0} \ldots n_{k}$ and applying the operator $d$ on each of the nodes $n_{1}, \ldots, n_{k}$ we have the identities:

$$
n_{i} n_{i+1}=n_{i} \cdot d n_{i+1}=d n_{i} \cdot n_{i+1}
$$

and hence

$$
n_{0} \ldots n_{k}=\left(n_{0} n_{1}\right) \cdot\left(n_{1} n_{2}\right) \cdots\left(n_{k-1} n_{k}\right)
$$

(concatenation of bonds, i.e. elementary strings)

$$
=\left(n_{0} d n_{1}\right) \cdot\left(n_{1} d n_{2}\right) \cdots\left(n_{k-1} d n_{k}\right)=n_{0} \cdot d n_{1} \cdot d n_{2} \cdots d n_{k}=: n_{0} d n_{1} \ldots d n_{k}
$$


Proof: The latter manipulations exploit the algebraic (concatenation) rules we have already established above (remember the definition of $d n_{i}$ as a certain superposition of 1-strings). The former identities hold because

$$
n_{i} \cdot d_{i+1}=n_{i} \cdot\left(\sum_{k}\left(d_{k(i+1)}-d_{(i+1) k}\right)\right)
$$

with $d_{k(i+1)}$ denoting the string $n_{k} n_{i+1}$ etc., hence $n_{i} \cdot d_{k(i+1)}=\delta_{i k} \cdot d_{i(i+1)}$. That is, it survives only the one term $d_{i(i+1)}$ or $n_{i} n_{i+1}$ since the other term $n_{i} \cdot d_{(i+1) k}$ is always zero by the definition of the concatenation product.

It is exactly these abstract (usually uninterpreted) objects $a_{0} d a_{1} \ldots d a_{k}$ which occur in the abstract formalism of, say, 'non-commutative de Rham complexes' or the 'universal differential algebra' (see e.g. [23] or for the case of discrete sets [26]).

\section{Remarks}

1. In an earlier version ([27]) we based most of the algebraic calculus on the concept and properties of tensor products (of e.g. modules) which, we think, has obscured a little bit the very suggestive geometric imagery. On the other side it is perhaps closer to the abstract framework of non-commutative geometry.

2. In the same paper we showed that our strings realize also the abstract structure of a 'groupoid'. For sake of brevity we omit the developement of the corresponding framework in this paper.

The above shows that our strings form a graded algebra with concatenation as multiplication. In a next step we show that the coboundary operator $d$ fulfills the so-called (graded) 'Leibniz rule' (wellknown from e.g. the exterior differential algebra)

3.11 Observation (Graded Leibniz Rule): With $w_{1}, w_{2}$ two strings, it holds

$$
d\left(w_{1} \cdot w_{2}\right)=d w_{1} \cdot w_{2}+(-1)^{\operatorname{deg}\left(w_{1}\right)} w_{1} \cdot d w_{2}
$$

where deg $\left(w_{1}\right)=$ number of edges in $w_{1}$

In the special case of zero-strings or functions from $C_{0}$ the analogous formula reads:

$$
d(f \cdot g)=d f \cdot g+f \cdot d g
$$

as $\operatorname{deg}(f)=0$.

Proof: Whereas the latter result is contained in the former one, we prefer to supply an extra proof as kind of a warm up.

$$
d(f \cdot g)=\sum_{i} f_{i} g_{i} d n_{i}=\sum_{i}\left(\sum_{k}\left(n_{k} n_{i}-n_{i} n_{k}\right)\right)
$$




$$
d f \cdot g+f \cdot d g=\left(\sum_{i, k} f_{i} g_{i}\left(n_{k} n_{i}\right)-\sum_{i, k} f_{i} g_{k}\left(n_{i} n_{k}\right)\right)+\left(\sum_{i, k} f_{k} g_{i}\left(n_{k} n_{i}\right)-\sum_{i, k} f_{i} g_{i}\left(n_{i} n_{k}\right)\right)
$$

The mixed $f_{i} g_{k}$-terms in the latter equation cancel each other (after a change of dummy variables), hence the result.

In the case of general strings with

$$
w_{1}=n_{i_{0}} \ldots n_{i_{k}}, w_{2}=n_{j_{0}} \ldots n_{j_{l}}
$$

we can split the action of $d$ on $w_{1} \cdot w_{2}$ in two groups of terms, the one comprising the action within $w_{1}$ or $w_{2}$, the other consisting of the terms which arise from the action of $d$ on the "interface" between $w_{1}$ and $w_{2}$.

The terms in the first group occur also in $d w_{1} \cdot w_{2}$ if $d$ acts within $w_{1}$ or in $w_{1} \cdot d w_{2}$ modified by an extra weight factor $(-1) \operatorname{deg}\left(w_{1}\right)$ if $d$ acts within $w_{2}$.

As to the interface-terms, there are three possibilities:

1. $n_{i_{k}}$ and $n_{j_{0}}$ are no nearest neighbors, i.e. they are more than one bond distance apart. In that case $w_{1} \cdot w_{2}=0$, hence $d\left(w_{1} \cdot w_{2}\right)=0$. On the other side, under this proviso no term in $d w_{1}$ is incident with $w_{2}$ and vice versa, that is $d w_{1} \cdot w_{2}=0=w_{1} \cdot d w_{2}$.

2. $n_{i_{k}} \neq n_{j_{0}}$ but they are nearest neighbors. This implies $w_{1} \cdot w_{2}=0=d\left(w_{1} \cdot w_{2}\right)$. Now there is exactly one term in $d w_{1}$ incident with $w_{2}$, namely $n_{i_{0}} \ldots n_{i_{k}} n_{j_{0}}$ carrying the weight $(-1)^{i_{k}+1}$. A corresponding term occurs in $d w_{2}$, carrying the weight $(-1)^{0}=1$. In the graded Leibniz rule this latter term (standing in front of $\left.w_{1} \cdot d w_{2}\right)$ is multiplied by $(-1)^{i_{k}}$, yielding the superposition

$$
(-1)^{i_{k}}\left((-1)^{1} \cdot(\text { string })+(-1)^{0} \cdot(\text { string })\right)=0
$$

3. The remaining possibility, $n_{i_{k}}=n_{j_{0}}$ is very simple. The corresponding end term in $d w_{1}$ leads away from $n_{i_{k}}=n_{j_{0}}$. So its concatenation with $w_{2}$ is zero. The same holds for the initial term in $d w_{2}$. That is, in this situation all the non-zero terms in $d w_{1} \cdot w_{2}, w_{1} \cdot d w_{2}$ are internal terms, falling in the first group, discussed above and for which the stated identit6y has already been verified.

This proves the graded Leibniz rule for strings (a different proof have been given in [28]).

We have now to explain why we call our algebra a 'semi-differential algebra'. To this end we show that in general, i.e. if the underlying graph is not 'complete' or a 'simplex', the relations $d \cdot d=0$ and $\delta \cdot \delta=0$ do not hold for every string or node. On the other side they are fulfilled for a complete graph. Phenomena like these are analyzed in more detail (among other things) by T.Nowotny in his Diploma thesis (28]) and are not difficult to prove anyhow in full generality. Therefore we content 
ourselves with giving the main clue of the reasoning (the phenomenon was already discussed in [27], section 4.3).

Take e.g. a graph, consisting of the nodes $n_{0}, n_{1}, n_{2}$ and the bonds $n_{0} n_{1}, n_{1} n_{2}$ (plus the "opposite bonds" $n_{1} n_{0}, n_{2} n_{1}$ ). We have

$$
d\left(n_{0}\right)=-n_{0} n_{1}+n_{1} n_{0} \quad \text { and } \quad d d\left(n_{0}\right)=-n_{0} n_{1} n_{2}+n_{2} n_{1} n_{0} \neq 0
$$

On the other hand, if the bonds $n_{0} n_{2}, n_{2} n_{0}$ were present we would have

$$
d n_{0}=-n_{0} n_{1}-n_{0} n_{2}+n_{2} n_{0}+n_{1} n_{0}
$$

and it is easy to see that all the terms in $d d n_{0}$ do in fact cancel.

Analyzing the general case a little bit more systematically we observe the following: In the 'reduced graph' (i.e. some bonds missing) the following can happen. Apply $d$ to a given string which yields e.g. an insertion between node $n_{i-1}$ and node $n_{i}$, e.g. $\ldots n_{i-1} n_{\nu} n_{i} \ldots$ with a weight $(-1)^{i}$. Applying $d$ again may yield another admissible insertion of the type $\quad \ldots n_{i-1} n_{\mu} n_{\nu} n_{i} \ldots$ coming with the weight $(-1)^{i} \cdot(-1)^{i}$ provided that $n_{\mu}$ is connected with $n_{i}$ and $n_{\nu}$.

On the other hand the "counterterm" with the weight $(-1)^{i} \cdot(-1)^{i+1}$ may be missing as it can happen that $n_{\mu}$ is connected with $n_{i-1}$ and $n_{\nu}$ but not with $n_{i-1}$ and $n_{i}$ so that $\ldots n_{i-1} n_{\mu} n_{i} \ldots$ does not show up in the first step in contrast to the analogous term with $n_{\nu}$. A similar result holds for $\delta$, hence:

3.12 Conclusion: We call the above algebra a 'semi-differential algebra' since in general (if some bonds are missing) $d d \neq 0, \delta \delta \neq 0$ on certain strings. If, on the other side, the underlying graph is complete, we have $d d=\delta \delta=0$

These last observations are perhaps of some help if one wants to compare our (more "bottom up") approach with a to some extent alternative (and more "top down") approach, which starts from the concept of the "universal differential algebra' (see the remarks at the beginning of this section). It is then an almost trivial observation (corresponding results being almost ubiquituous in abstract algebra) that every 'differential algebra' is the homomorphic image of the universal one; put differently: it is isomorphic to the universal algebra divided by a certain '(differential) ideal' (see below). These results have then been aplied to discrete sets by e.g. Dimakis,MuellerHoissen et al (26]).

On the other hand we want to stress that models, in our case networks or graphs, tend to have a lot more interesting geometric or algebraic structure than suggested by such an abstract viewpoint and should be analyzed for their own sake (see also the following subsections). We want now to briefly comment on one particular feature one should at least be aware of if one favors the abstract approach. We would like to call it the 'problem of unnatural relations'.

To conform with the more abstract notation adopted in the above mentioned literature (or [27]), we now denote the universal differential algebra over a given 
set of nodes (i.e. the complete graph) by $\Omega^{u}=\sum \Omega_{k}^{u}$, the reduced algebra (i.e. the actually given graph with some bonds missing in general) by $\Omega=\sum \Omega_{k}$. Furthermore, $d_{u}$ is the differential on $\Omega^{u}$ with $d_{u} \cdot d_{u}=0$. Note that in $\Omega_{k}^{u}$ each sequence of $(k+1)$ nodes is an admissible string.

We can now define a projector $\Pi$ which projects $\Omega^{u}$ onto $\Omega$ by:

$$
\Pi\left(n_{0} \ldots n_{k}\right)=0
$$

if $\left(n_{0}, \ldots, n_{k}\right)$ is not admissible in $\Omega$

$$
\Pi\left(n_{0} \ldots n_{k}\right)=n_{0} \ldots n_{k}
$$

if $\left(n_{0}, \ldots, n_{k}\right)$ is admissible.

3.13 Consequence: We have

$$
\Pi=\Pi^{2}, \Omega^{u}=\Pi \Omega^{u}+(\mathbb{I}-\Pi) \Omega^{u}
$$

with $\Pi \Omega^{u}=\Omega$. We could now continue by defining

$$
d:=\Pi \circ d_{u} \circ \Pi
$$

which leaves $\Omega$ invariant but in general $d \cdot d \neq 0$ in contrast to $d_{u} \cdot d_{u}=0$ as we have seen above.

We observe that $\operatorname{Ker}(\Pi)$ is a two-sided ideal $I$ consisting of the elements $n_{0 \ldots k}$ having at least one pair of consecutive nodes not being connected by a bond in the reduced graph. However, this ideal $I$ is not left invariant under the action of $d_{u}$ ! A closer analysis shows that $d_{u}\left(n_{0 \ldots k}\right) \notin I$ if $d_{u}$ creates 'insertions' between non-connected neighbors in the reduced graph s.t. non-admissible elements become admissible, i.e. connected. We hence have:

3.14 Observation: In general there exist elements $n_{0 \ldots k} \in \operatorname{Ker}(\Pi)$ s.t. $\Pi\left(d_{u}\left(n_{0 \ldots k}\right)\right) \neq$ 0 , in other words, I is in general not left invariant by $d_{u}$.

If one wants to make $\Omega$ into a real differential algebra one has to enlarge I!

3.15 Consequence: The ideal $I^{\prime}=I+d_{u} \circ I$ is invariant under $d_{u}$ and $d$ defines a differential algebra on the smaller algebra $\Omega^{u} / I^{\prime} \subset \Omega$ with $\Omega=\Omega^{u} / \operatorname{Ker}(\Pi)$.

(That $I^{\prime}$ is an ideal left invariant by $d_{u}$ is easy to prove with the help of the property $\left.d_{u} \cdot d_{u}=0\right)$.

So much so good, but in our view there now emerges a certain problem. $\Omega^{u} / I^{\prime}$ is the algebra one automatically arrives at if one defines the homomorphism $\Phi$ from $\Omega^{u}$ to the reduced differential algebra in the following canonical way:

$$
\Phi: n_{i} \rightarrow n_{i} \quad d_{u} n_{i} \rightarrow d n_{i}
$$


i.e. under the premise that $d$ defines already another differential algebra. It is in this restricted sense the above mentioned general result has to be understood.

On the other hand this may (and in general: will) lead to a host of, at least in our view, very unnatural relations in concrete models like e.g. our network, models which may already carry a certain physically motivated interpretation going beyond being a mere example of an abstract differential algebra. Note e.g. that in our algebra $\Omega$ an element like $n_{123}$ is admissible (i.e. non-zero) if $n_{1}, n_{2}$ and $n_{2}, n_{3}$ are connected. $n_{123}$ may however arise from a differentiation process (i.e. from an insertion) like $d_{u}\left(n_{13}\right)$ with $n_{1}, n_{3}$ not connected.

This is exactly the situation discussed above:

$$
n_{13} \in I \quad \text { but } \quad d_{u}\left(n_{13}\right) \notin I
$$

Dividing now by $I^{\prime}$ maps $d_{u}\left(n_{13}\right)$ onto zero whereas there may be little physical or geometric reason for $n_{123}$ or a certain combination of such admissible elements being zero in our network.

3.16 Conclusion: Given a concrete physical network $\Omega$ one has basically two choices. Either one makes it into a full-fledged differential algebra by imposing further relations which may however be unnatural from a physical point of view and very cumbersome for complicated networks. This was the strategy e.g. followed in [20].

Or one considers $\Omega$ as the fundamental object and each admissible element in it being non-zero. As a consequence the corresponding algebraic/differential structure on $\Omega$ may be less smooth at first glance ( $d d \neq 0$ in general), but on the other side considerably more natural! At the moment we refrain from making a general judgement about the respective advantages of these two different approaches whereas we would probably prefer the latter one.

\subsection{The Graph as a (Higher) Simplicial Complex}

In the following we will change our geometric point of view to some extent. In the previous subsection the geometric building blocks have been strings or walks, that is, more or less one-dimensional objects. Now we will be concerned with in some sense higher dimensional patterns. To this end we start from a fixed graph $G$ with vertex set $V$ and edge set $E$. In the literature graphs are frequently considered as so-called 'one-dimensional simplicial complexes' with the nodes as 0-simplices and the bonds as 1-simplices. We think this point of view is unnecessarily restrictive (while there exist of course certain mathematical reasons for this restriction we do not mention). Having our own working philosophy of networks as models for microscopic space-time in mind, this concept can and should be generalized.

3.17 Definition (Subsimplices): We call a subset of $(k+1)$ vertices a ' $k$-simplex', $s_{k}$, or 'complete subgraph' if every two nodes of $s_{k}$ are connected by a bond. 
3.18 Observation: If the node degree, $v_{i}$, of $n_{i}$ is smaller than $\infty$ then $n_{i}$ can lie in at most a finite set of different simplices, an upper bound being provided by the number of different subsets of bonds emerging from $n_{i}$

Proof: Assume that $s_{k}, s_{l}$ are two different simplices containing $n_{i}$. By definition $n_{i}$ is linked with all the other nodes in $s_{k}$ or $s_{l}$. As these sets are different by assumption, the corresponding subsets of bonds emerging from $n_{i}$ are different. On the other side, not every subset of such bonds corresponds to a simplex (there respective endpoints need not form a simplex), which proves the upper bound stated above.

\subsection{Consequence:}

1. The set of subsimplices is evidently 'partially ordered' by inclusion

2. Furthermore, if $s$ is a simplex, each of its subsets is again a simplex (called a 'face')

3. It follows from Observation 3.18 that each of these 'chains' of linearly ordered simplices is finite with the same upper bound as in Observation 3.18. In other words each chain has a maximal element, a so-called 'maximal subsimplex', abbreviated by mss. By the same token each node lies in at least one (but generically several) mss

4. Such a mss with $n_{i}$ being a member can comprise at most $\left(v_{i}+1\right)$ nodes, in other words, its cardinality is the minimum of these numbers when $n_{i}$ varies over the mss.

Remark: Such mss are called in combinatorics or graph theory 'cliques'. Their potential physical role as building blocks of the micro-structure of space-time in our particular approach has been discussed in greater depth in [11].

3.20 Observation: The class of simplices, in particular the mss, containing a certain fixed node, $n_{i}$, can be generated in a completely algorithmic way, starting from $n_{i}$. The first level consists of the bonds with $n_{i}$ an end node, the second level comprises the triples of nodes ('triangles'), $\left(n_{i} n_{k} n_{l}\right)$, with the nodes linked with each other and so forth. Each level set can be constructed from the preceding one and the process stops when a mss is reached.

Remark: Note that at each intermediate step, i.e. having already constructed a certain particular subsimplex, one has in general several possibilities to proceed. On the other hand, a chain of such choices may differ at certain places from another one but may lead in the end to the same simplex (being simply a permutation of the nodes of the latter simplex). 
In a next step we can give the simplices an 'orientation'.

3.21 Definition (Orientation): The above simplices can be oriented via

$$
n_{\pi(0)} \ldots n_{\pi(k)}=\operatorname{sgn}(\pi) \cdot n_{0} \ldots n_{k}
$$

$\pi$ being a permutation of $(0, \ldots, k)$

This definition can be used to give the set of simplices an algebraic structure by identifying the simplices which fall in the same equivalence class with respect to $\operatorname{sgn} \pi$. The respective equivalence class will henceforth be denoted by $\left(n_{i_{0}} \ldots n_{i_{k}}\right)$ and we make these classes into an orthonormal basis of some vector space of, say, finite sums over e.g. $\mathbb{C}$. The other class (negative orientation) is then simply the opposite vector $-\left(n_{i_{0}} \ldots n_{i_{k}}\right)$.

3.22 Consequence: The above observations show that our set of oriented simplices establish what is called in algebraic topology an 'abstract' or 'combinatorial' 'simplicial complex'. If the node degree is uniformly bounded away from $\infty$ on $V$, this simplicial complex has a 'finite degree' (which is the cardinality of the largest occurring mss).

Remark: By means of an elegant argument one can show that such a simplicial complex with degree, say, $n$ can always be embedded in $\mathbb{R}^{2 n+1}$ (see e.g. [30]).

On this simplicial complex we can now define two operators $\delta, d$ which, however, differ from the ones defined in subsection 3.1. We will show in particular that $\delta \cdot \delta=$ $d \cdot d=0$ always holds.

3.23 Definition/Observation: The 'boundary operator' $\delta$ acts in the following way on the basis elements:

$$
\delta\left(n_{0} \ldots n_{k}\right):=\sum_{l=0}^{k}(-1)^{l} \cdot\left(n_{0} \ldots \widehat{n_{l}} \ldots n_{k}\right)
$$

with $\widehat{n_{l}}$ being omitted as usual. Note that the terms on the rhs are again simplices (faces). It is a standard procedure to prove that $\delta \cdot \delta=0$ holds. The 'coboundary operator' $d$ is defined via:

$$
d\left(n_{0} \ldots n_{k}\right):=\sum_{n_{\nu}}\left(n_{\nu} n_{0} \ldots n_{k}\right)
$$

the sum on the rhs running over the nodes $n_{\nu}$ so that the terms under the sum form again $(k+1)$-simplices (or, put differently, the sum extends over all nodes with terms set to zero if they do not form $(k+1)$-simplices). It holds $d \cdot d=0$.

Remark: Note that in the above formula it is important to work with equivalence 
classes. Otherwise the definition would become quite cumbersome. As in the case of 'exterior algebra' of e.g. forms the ordering in (...) is 'anticommutative', that is,

$$
\left(n_{\nu} n_{0} \ldots n_{k}\right)=-\left(n_{0} n_{\nu} \ldots n_{k}\right)
$$

Proof: We show that $d \cdot d=0$ in fact holds. We have:

$$
d d(s)=\sum_{\mu} \sum_{\nu}\left(n_{\mu} n_{\nu} s\right)
$$

with $s$ a certain simplex. As both $\left(n_{\nu} n_{\mu} s\right)$ and $\left(n_{\mu} n_{\nu} s\right)=-\left(n_{\nu} n_{\mu} s\right)$ occur in the double sum all the terms on the rhs cancel.

3.24 Observation: If we consider the $s_{k}$ as basis elements of the vector space introduced above, it follows that $d$ is the adjoint of $\delta$.

Proof:

$$
<s_{k}\left|\delta s_{k+1}^{\prime}>=\sum_{\nu=0}^{k+1}(-1)^{\nu} \cdot<s_{k}\right|\left(n_{0}^{\prime} \ldots \widehat{n_{\nu}^{\prime}} \ldots n_{k+1}^{\prime}\right)>
$$

We see that at most one non-zero term can occur on the rhs, say, $(-1)^{\nu}<s_{k} \mid \pm s_{k}>$, \pm depending on the respective orientation. On the other side,

$$
<d s_{k}\left|s_{k+1}^{\prime}>=\sum_{n_{\nu}}<\left(n_{\nu} n_{0} \ldots n_{k}\right)\right|\left(n_{0}^{\prime} \ldots n_{k+1}^{\prime}\right)>
$$

Again there exists at most one non-zero term on the rhs with the node sequence on the left side of the scalar product being a certain permutation of the node sequence on the right side. In a first step we permute the $n_{0} \ldots n_{k}$ on the left side so that they are standing in the same relative order as on the right side. This yields the same prefactor \pm 1 as in the formula above. We then commute the additional node $n_{\nu}$ until it occupies the same position as in $s_{k+1}^{\prime}$ yielding another prefactor $(-1)^{\nu}$

Remark: This latter version is perhaps closer to the original point of view adopted in cohomology theory (in the sense of, say, Alexander), where cotheory typically lives on 'dual spaces' (cf. e.g. 29]).

3.25 Corollary: The above notion of orientation shows that e.g. the 1-simplices (i.e. bonds) correspond rather to the oriented bonds $b_{i k}=n_{i} n_{k}-n_{k} n_{i}$ than to the directed bonds $d_{i k}=n_{i} n_{k}$ introduced in section 3.1 (see e.g. item 3 of Definition 3.1). The definition of $d$, given in this subsection, can then be rewritten as

$$
d\left(n_{0}\right)=\sum_{n_{k}}\left(n_{k} n_{0}\right)=\sum_{k} b_{k 0}
$$

hence turns out to be consistent with the previous one given in subsection 3.1 at least on the first level. 
On the higher levels the correspondence is slightly trickier, see the following remark.

Remark: For higher simplices the correspondence is slightly more involved. Take e.g. a triangle $\left(n_{0} n_{1} n_{2}\right)$. In the spirit of subsection 3.1 one can take all the possible 3 -strings which can be built over this simplex, in other words, all the possible permutations of $n_{0} n_{1} n_{2}$ and define the algebraic, oriented simplex $\left(n_{0} n_{1} n_{2}\right)$ of subsection 3.2 as follows:

$$
\left(n_{0} n_{1} n_{2}\right):=\sum_{p e r} \operatorname{sgn}(\text { per }) \cdot n_{i_{0}} n_{i_{1}} n_{i_{2}}
$$

with the sum running over all the permutations of $(0,1,2)$ and the terms on the rhs being strings in the sense of subsection 3.1. It follows directly that the sum is anticommutative with respect to rearrangements of the nodes (it may be convenient to add a global combinatorial factor $(k+1) !^{-1}$ on the rhs ). From this point of view the correspondence is reminiscent of the way exterior forms are built from tensors.

\subsection{Yet another version of Discrete Calculus}

In this last subsection we briefly comment on yet another version of discrete calculus, which is, at first glance, perhaps the most natural one and which is absent in approaches starting from the abstract universal differential algebra. As this variant is developed further in the diploma thesis of T.Novotny (some results can already be found in [11]), in particular concerning the method of 'discrete Euler-LagrangeVariation', we will not elaborate very much on this point.

\subsection{Definition (Partial Forward Derivative at Node (i)):}

$$
\nabla_{i k} f(i):=f(k)-f(i)
$$

where $n_{i}, n_{k}$ are 'nearest-neighbor-nodes', i.e. being connected by a bond.

\subsection{Observation:}

$$
\begin{aligned}
\nabla_{i k}(f \cdot g)(i) & =(f \cdot g)(k)-(f \cdot g)(i) \\
& =\nabla_{i k} f(i) \cdot g(i)+f(k) \cdot \nabla_{i k} g(i) \\
& =\nabla_{i k} f(i) g(i)+f(i) \nabla_{i k} g(i)+\nabla_{i k} f(i) \nabla_{i k} g(i)
\end{aligned}
$$

In other words the "derivation" $\nabla$ does not obey the ordinary Leibniz rule. In fact, application of $\nabla$ to, say, higher powers of $f$ becomes increasingly cumbersome (nevertheless there is a certain systematic in it). One gets for example (with $q:=\nabla_{i k}$ ):

$$
q\left(f_{1} \cdots f_{n}\right)=\sum_{i} f_{1} \cdots q\left(f_{i}\right) \cdots f_{n}+\sum_{i j} f_{1} \cdots q\left(f_{i}\right) \cdots q\left(f_{j}\right) \cdots f_{n}+\ldots+q\left(f_{1}\right) \cdots q\left(f_{n}\right)
$$


Due to the discreteness of the formalism and, as a consequence, the inevitable bilocality of the derivative there is no chance to get something like a true Leibniz rule on this level.

It is perhaps worth mentioning that the above establishes an interesting abstract algebraic multiplication structure which is called in 25] a 'Cuntz algebra structure' (occurring there however in another context).

\subsection{Observation (Cuntz algebra):}

$$
q(f \cdot g)=q(f) \cdot g+f \cdot q(g)+q(f) \cdot q(g)
$$

and analogously for vector fields $\sum a_{i k} \nabla_{i k}$.

With $u:=1+q$ we furthermore get:

$$
u(f \cdot g)=u(f) \cdot u(g)
$$

and

$$
q(f \cdot g)=q(f) \cdot g+u(f) \cdot q(g)
$$

i.e. a 'twisted derivation' with $u$ an endomorphism on some algebra, $\mathcal{A}$, of functions on $V$.

As is the case with $\nabla_{i k}$, the product rule for higher products can be inferred inductively:

$q\left(f_{1} \cdots f_{n}\right)=\sum_{i} f_{1} \cdots q\left(f_{i}\right) \cdots f_{n}+\sum_{i j} f_{1} \cdots q\left(f_{i}\right) \cdots q\left(f_{j}\right) \cdots f_{n}+\ldots+q\left(f_{1}\right) \cdots q\left(f_{n}\right)$

3.29 Definition ((Co)Tangential Space): We call the space spanned by the $\nabla_{i k}$ at node $n_{i}$ the tangential space $T_{i}$. Correspondingly we introduce the space spanned by the $d_{i k}$ at node $n_{i}$ and call it the cotangential space $T_{i}^{*}$ with the $d_{i k}$ acting as linear forms over $T_{i}$ via:

$$
<d_{i k} \mid \nabla_{i j}>=\delta_{k j}
$$

3.30 Definition/Observation: Higher tensor products of differential forms at a node $n_{i}$ can now be defined as multilinear forms:

$$
<d_{i k_{1}} \otimes \cdots \otimes d_{i k_{n}} \mid\left(\nabla_{i l_{1}}, \cdots, \nabla_{i l_{n}}\right)>:=\delta_{k_{1} l_{1}} \times \cdots \times \delta_{k_{n} l_{n}}
$$

and linear extension.

In a next step we extend these concepts to functions $f \in C_{0}$ and 'differential operators' or 'vector fields' $\sum a_{i k} \nabla_{i k}$. We have to check whether this is a natural(!) 
definition.

3.31 Observation: Vector fields $v:=\sum a_{i k} \nabla_{i k}$ are assumed to act on functions $f=\sum f_{i} n_{i}$ in the following manner:

$$
v(f):=\sum a_{i k}\left(f_{k}-f_{i}\right) n_{i}
$$

i.e. they $\operatorname{map} C_{0} \rightarrow C_{0}$.

3.32 Corollary: Note that this implies:

$$
\begin{aligned}
& \nabla_{i k} n_{k}=n_{i} \quad \nabla_{i k} n_{i}=-n_{i} \\
& \nabla_{k i} n_{k}=-n_{k} \quad \nabla_{k i} n_{i}=n_{k}
\end{aligned}
$$

3.33 Observation: 'Differential forms' $\omega=\sum g_{i k} d_{i k}$ act on vector fields $v=$ $\sum a_{i k} \nabla_{i k}$ according to:

$$
<\omega \mid v>=\sum g_{i k} a_{i k} n_{i}
$$

With these definitions we can calculate $<d f \mid v>$ with

$$
d f=\sum\left(f_{k}-f_{i}\right) d_{i k}
$$

Hence:

$$
<d f\left|v>=<\sum\left(f_{k}-f_{i}\right) d_{i k}\right| \sum a_{i k} \nabla_{i k}>=\sum\left(f_{k}-f_{i}\right) a_{i k} n_{i}
$$

which equals:

$$
\left(\sum a_{i k} \nabla_{i k}\right)\left(\sum f_{i} n_{i}\right)=v(f)
$$

3.34 Consequence: Our geometric interpretation of the algebraic objects reproduces the relation:

$$
<d f \mid v>=v(f)
$$

known to hold in ordinary differential geometry, as is the case for the following relations, and shows that the definitions made above seem to be natural. Furthermore, vector and covector fields are left modules under the action of $\mathcal{A}$ :

$$
\begin{aligned}
\left(\sum f_{i} n_{i}\right)\left(\sum a_{i k} \nabla_{i k}\right) & :=\sum f_{i} a_{i k} \nabla_{i k} \\
\left(\sum f_{i} n_{i}\right)\left(\sum g_{i k} d_{i k}\right) & :=\sum f_{i} g_{i k} d_{i k}
\end{aligned}
$$


3.35 Conclusion: The above shows that, in contrast to classical differential geometry, we have a dual pairing between vector and covector fields with the vector fields acting as 'twisted' derivations on the node functions while the corresponding differential forms obey the graded Leibniz rule like their classical counterparts.

Another important geometrical concept is the notion of 'connection' or 'covariant derivative'. Starting from the abstract concept of (linear) connection in the sense of Koszul it is relatively straightforward to extend this concept to the non-commutative situation, given a 'finite projective module' over some algebra $\mathcal{A}$ (instead of the sections of a vector bundle over some manifold $\mathcal{M}$, the role of $\mathcal{A}$ being played by the functions over $\mathcal{M}$; see e.g. [23] or [25], as to various refinements and improvements cf. e.g. [31] and further references given there).

Without going into any details we want to briefly sketch how the concept of connection can be immediately implemented in our particular model without referring to the more abstract work. We regard (in a first step) a connection as a (linear) map from the fields of tangent vectors to the tensor product of tangent vectors and dual differential forms as defined above (and having certain properties).

3.36 Definition/Observation(Connection): A field of connections, $\Gamma$, is defined at each node $n_{i}$ by a (linear) map:

$$
\nabla_{i k} \rightarrow \gamma_{k l}^{j}\left(n_{i}\right) \cdot \nabla_{i j} \otimes d_{i}^{l}
$$

where the index $i$ plays rather the role of the "coordinate" $n_{i}$, the index $l$ is raised in order to comply with the summation convention. The $\gamma_{k l}^{j}$ 's are called 'connection coefficients'. The corresponding 'covariant derivative' $\nabla$ obeys the relations:

i)

$$
\nabla(v+w)=\nabla(v)+\nabla(w)
$$

ii)

$$
\nabla(f \cdot v)=v \otimes d f+\nabla(v) \cdot f
$$

iii)

$$
\nabla\left(\nabla_{i k}\right)=\Gamma\left(\nabla_{i k}\right) \quad d f=\sum\left(f_{k}-f_{i}\right) d_{i k}
$$

Remark: The tensor product in ii) is understood as the pointwise product of fields at each node $n_{i}$, i.e. $\nabla_{i k}$ going with $d_{i k}$. This is to be contrasted with the abstract notion of tensor product in e.g. the above differential algebra $\Omega(\mathcal{A})$ which does not act locally, the space consisting of, say, elements of the kind $n_{1} \otimes n_{2} \otimes \cdots \otimes n_{k}$. These different parallel structures over the same model shall be scrutinized in more detail elsewhere. Note that the above extra locality structure is a particular property of our model class and does not (openly) exist in the general approach employing arbitrary projective modules respectively differential algebras. 


\section{Intrinsic Dimension in Networks, Graphs and other Discrete Systems}

There exist a variety of concepts in modern mathematics which extend the ordinary or naive notion of dimension one is accustomed to in e.g. differential topology or linear algebra. In fact, topological dimension and related concepts are notions which are perhaps even closer to the underlying intuition (cf. e.g. [32]).

Apart from the purely geometric concept there is also an important physical role being played by something like dimension, having e.g. pronounced effects on the behavior of, say, many-body-systems near their phase transition points or in the critical region.

But even in the case of e.g. lattice systems they are usually treated as being embedded in an underlying continuous background space (typically euclidean) which supplies the concept of ordinary dimension so that the intrinsic dimension of the discrete array itself does usually not openly enter the considerations.

Anyway, it is worthwhile even in this relatively transparent situation to have a closer look on the situations where attributes of something like dimension really enter the physical stage. Properties of models of, say, statistical mechanics are typically derived from the structure of the microscopic interactions of their constituents. This then is more or less the only place where dimensional aspects enter the calculations.

Naive reasoning might suggest that it is something like the number of nearest neighbors (in e.g. lattice systems) which encodes the dimension of the underlying space and influences via that way the dynamics of the system. However, this surmise, as we will show in the following, does not reflect the crucial point which is considerably more subtle.

This holds the more so for systems which cannot be considered as being embedded in a smooth regular background and hence do not inherit their dimension from the embedding space. A case in point is our primordial network in which Planck-scalephysics is assumed to take place. In our approach it is in fact exactly the other way round: Smooth space-time is assumed to emerge via a phase transition or a certain cooperative behavior and after some 'coarse graining' from this more fundamental structure.

4.1 Problem: Formulate an intrinsic notion of dimension for model theories without making recourse to the dimension of some continuous embedding space.

In a first step we will show that graphs and networks as introduced in the preceding sections have a natural metric structure. We have already introduced a certain neighborhood structure in a graph with the help of the minimal number of consecutive bonds connecting two given nodes.

In a connected graph any two nodes can be connected by a sequence of bonds. Without loss of generality one can restrict oneself to paths. One can then define the 
length of a path (or sequence of bonds) by the number $l$ of consecutive bonds making up the path.

4.2 Observation/Definition: Among the paths connecting two arbitrary nodes there exists at least one with minimal length which we denote by $d\left(n_{i}, n_{k}\right)$. This $d$ has the properties of a metric, i.e:

$$
\begin{aligned}
d\left(n_{i}, n_{i}\right) & =0 \\
d\left(n_{i}, n_{k}\right) & =d\left(n_{k}, n_{i}\right) \\
d\left(n_{i}, n_{l}\right) & \leq d\left(n_{i}, n_{k}\right)+d\left(n_{k}, n_{l}\right)
\end{aligned}
$$

(The proof is more or less evident).

4.3 Corollary: With the help of the metric one gets a natural neighborhood structure around any given node, where $\mathcal{U}_{m}\left(n_{i}\right)$ comprises all the nodes, $n_{k}$, with $d\left(n_{i}, n_{k}\right) \leq m$, $\partial \mathcal{U}_{m}\left(n_{i}\right)$ the nodes with $d\left(n_{i}, n_{k}\right)=m$.

This natural neighborhood structure enables us to develop the concept of an intrinsic dimension on graphs and networks. To this end one has at first to realize what property really matters physically (e.g. dynamically), independently of the kind of model or embedding space.

4.4 Observation: The crucial and characteristic property of, say, a graph or network which may be associated with something like dimension is the number of 'new nodes' in $\mathcal{U}_{m+1}$ compared to $\mathcal{U}_{m}$ for $m$ sufficiently large or $m \rightarrow \infty$. The deeper meaning of this quantity is that it measures the kind of 'wiring' or 'connectivity' in the network and is therefore a 'topological invariant'.

Remark: In the following I shall be very brief on this ramified topic as much more details have been presented and proved in a separate paper ([8]) to which the interested reader is referred. Instead of that I would like to discuss certain aspects and ideas being related to this concept.

In many cases one expects the number of nodes in $\mathcal{U}_{m}$ to grow like some power $D$ of $m$ for increasing $m$. By the same token one expects the number of new nodes after an additional step to increase proportional to $m^{D-1}$. With $|\cdot|$ denoting the number of nodes we hence expect frequently the following to hold:

$$
\left|\mathcal{U}_{m+1}\right|-\left|\mathcal{U}_{m}\right|=\left|\partial \mathcal{U}_{m+1}\right|=f(m)
$$

with

$$
f(m) \sim m^{D-1}
$$

for $m$ large. 
4.5 Definition: "The" intrinsic dimension D of a homogeneous (infinite) graph is given by

$$
D-1:=\lim _{m \rightarrow \infty}(\ln f(m) / \ln m)
$$

or

$$
D:=\lim _{m \rightarrow \infty}\left(\ln \left|\mathcal{U}_{m}\right| / \ln m\right)
$$

provided that a unique limit exists!

What does exist in any case is lim inf respectively lim sup which can then be considered as upper and lower dimension. If they coincide we are in the former situation. By 'homogeneous' we mean that the graph "looks locally more or less the same" geometrically everywhere, in particular its dimension $D$ should not depend on the reference point (and that the two definitions of graph dimension given above coincide!).

Remarks:

1. One might naively expect that 'regularity', i.e. constant node degree, plus certain other conditions imply homogeneity but this is a highly non-trivial question. There are e.g. simple examples of regular graphs which do not "look the same" around every node. That is, a so-called 'trivalent graph', which is frequently taken as a discretized standard example in various versions of quantum gravity (see e.g. the above mentioned literature about spin networks), is 'planar', i.e. can be embedded in the plane. This may lead to the erroneous conclusion that its "natural dimension" is two. On the other side, our analysis shows, that its "intrinsic dimension" depends crucially on the details of its wiring and can even become infinite in case of a trivalent tree. Furthermore, it is a fascinating and non-trivial task to characterize e.g. the dimension of regular lattices (or 'triangulations') in a purely combinatorial manner without using a background space. This shall however be done elsewhere.

2. On the other side we showed in [8] that, among other things, uniform boundedness of the node degree guarantees already the independence of $D$ with respect to the reference point! Furthermore we were able to construct graph models for each given (fractal) dimension $D \in \mathbb{R}$. Due to discreteness, the relation between the two definitions of dimension may be a little bit subtle (as is the case for the various fractal dimensions; there may be exceptional graphs where they do not coincide!).

3. Other (but related) definitions of dimension are possible, incorporating e.g. the bonds instead of the nodes.

4. For practical purposes one may also introduce a notion of local dimension around certain nodes or within certain regions of a regular graph if the above limit is approached sufficiently fast locally. 
5. Particularly interesting phenomena are expected to show up if this concept is applied within the regime of 'random graphs' (see e.g. [11])

6. For some remarks concerning more or less related ideas being scattered in the literature (known to us) see 8]

That this definition is reasonable can be seen by applying it to ordinary cases like regular translation invariant lattices. It is however not evident that such a definition makes sense for arbitrary graphs, in other words, a (unique) limit point may not always exist. It seems to be a highly non-trivial task to characterize the conditions which imply such a limit to exists.

4.6 Observation For regular lattices D coincides with the dimension of the euclidean embedding space $D_{E}$.

Proof: This can be rigorously proved but it is perhaps more instructive to simply draw a picture of the consecutive series of neighborhoods of a fixed node for e.g. a 2-dimensional Bravais lattice. It is fairly obvious that for $\mathrm{m}$ sufficiently large the number of nodes in $\mathcal{U}_{m}$ goes like a power of $m$ with the exponent being the embedding dimension $D_{E}$ as the euclidean volume of $\mathcal{U}_{m}$ grows with the same power.

\section{Remarks:}

1. For $\mathcal{U}_{m}$ too small the number of nodes may deviate from an exact power law which in general becomes only correct for sufficiently large $m$.

2. The number of nearest neighbors, on the other side, does not influence the exponent but rather shows up in the prefactor. In other words, it influences $\left|\mathcal{U}_{m}\right|$ for $m$ small but drops out asymptotically by taking the logarithm. For an ordinary Bravais lattice with $N_{C}$ the number of nodes in a unit cell one has asymptotically:

$$
\begin{gathered}
\left|\mathcal{U}_{m}\right| \sim N_{C} \cdot m^{D_{E}} \quad \text { and hence: } \\
D=\lim _{m \rightarrow \infty}\left(\ln \left(N_{C} \cdot m^{D_{E}}\right) / \ln m\right)=D_{E}+\lim _{m \rightarrow \infty}\left(N_{C} / \ln m\right)=D_{E}
\end{gathered}
$$

independently of $N_{C}$.

Matters become much more interesting and subtle if one studies more general graphs than simple lattices. Note that there exists a general theorem (in fact a specialisation of the more general result about simplicial complexes we mentioned in the preceeding section to one-simplices), showing that practically every graph can be embedded in $\mathbb{R}^{3}$ and still quite a few in $\mathbb{R}^{2}$ (planar graphs).

This shows again that something like the dimension of the embedding space is in general not a characteristic property of a network or graph. Quite to the contrary, 
it is generically entirely uncorrelated with its intrinsic dimension we defined above. An extreme example is a 'tree graph', i.e. a graph without 'loops'. In the following we study an infinite, regular tree graph with node degree 3, i.e. 3 bonds branching off each node. The absence of loops means that the 'connectivity' is extremely low which results in an exceptionally high 'dimension' as we will see.

Starting from an arbitrary node we can construct the neighborhoods $\mathcal{U}_{m}$ and count the number of nodes in $\mathcal{U}_{m}$ or $\partial \mathcal{U}_{m} . \mathcal{U}_{1}$ contains 3 nodes which are linked with the reference node $n_{0}$. There are 2 other bonds branching off each of these nodes. Hence in $\partial \mathcal{U}_{2}=\mathcal{U}_{2} \backslash \mathcal{U}_{1}$ we have $3 \cdot 2$ nodes and by induction:

$$
\left|\partial \mathcal{U}_{m+1}\right|=3 \cdot 2^{m}
$$

which implies

$$
D-1:=\lim _{m \rightarrow \infty}\left(\ln \left|\partial \mathcal{U}_{m+1}\right| / \ln m\right)=\lim _{m \rightarrow \infty}(m \cdot \ln 2 / \ln m+3 / \ln m)=\infty
$$

Hence we have:

4.7 Observation(Regular Tree): The intrinsic dimension of an infinite regular tree is $\infty$ and the number of new nodes grows exponentially like some $n(n-1)^{m}$ (with $n$ being the node degree).

Remark: $D=\infty$ is mainly a result of the absence of loops and of regularity, in other words: there is exactly one path, connecting any two nodes. This is usually not so in other graphs, e.g. lattices, where the number of new nodes grows at a much slower pace (whereas the number of nearest neighbors can nevertheless be large). This is due to the existence of many loops s.t. many of the nodes which can be reached from, say, a node of $\partial \mathcal{U}_{m}$ by one step are already contained in $\mathcal{U}_{m}$ itself. On the other side we recently constructed ([8]) tree graphs having an arbitrary fractal dimension (they are however not regular, i.e. having a varying node degree, in particular they have a lot of end points). Hence the absence of loops is not the only reason for large graph dimensions.

We have seen that for, say, lattices the number of new nodes grows like some fixed power of $m$ while for, say, regular trees $m$ occurs in the exponent. The borderline can be found as follows:

4.8 Observation: If for $m \rightarrow \infty$ the average number of nodes in $\mathcal{U}_{m+1}$ per node contained in $\mathcal{U}_{m}$ is uniformly away from zero or, stated differently:

$$
\left|\mathcal{U}_{m+1}\right| /\left|\mathcal{U}_{m}\right| \geq 1+\varepsilon
$$

for some $\varepsilon \geq 0$ then we have exponential growth, in other words, the intrinsic dimension is $\infty$. 
The corresponding result holds with $\mathcal{U}_{m}$ being replaced by $\partial \mathcal{U}_{m}$.

Proof: If the above estimate holds for all $m \geq m_{0}$ we have by induction:

$$
\left|\mathcal{U}_{m}\right| \geq\left|\mathcal{U}_{m_{0}}\right| \cdot(1+\varepsilon)^{m-m_{0}}
$$

Potential applications of this concept of intrinsic dimension are manifold. Our main goal is it to develop a theory which explains how our classical space-time and what we like to call the 'physical vacuum' has emerged from a more primordial and discrete background via some sort of phase transition (the first preliminary steps being done in [11]).

In this context we can also ask in what sense macroscopic space-time dimension 4 is exceptional, i.e. whether it is merely an accident or whether there is a cogent reason for it. We hope or expect that the above concept of intrinsic dimension together with the dynamics of its possible change may help to come to grips with such a fundamental question.

\section{References}

[1] L.Smolin: "The Future of Spin Networks", gr-qc/9702030

[2] C.Rovelli: "Loop Quantum Gravity", gr-qc/9710008

[3] C.J.Isham: "Structural Issues in Quantum Gravity", gr-qc/9510063 (Lecture given at the GR14 Conference, Florence 1995)

[4] R.Penrose in: "Quantum Theory and Beyond", ed. T.Bastin, Cambridge Univ.Pr., Cambridge 1971, or

"Magic without Magic, John Archibald Wheeler", ed. J.R.Klauder, Freeman, San Francisco 1972

[5] L.Smolin in: "Conceptual Studies of Quantum Gravity", eds. A.Ashtekar, J.Stachel, Einstein Studies Vol.2, Birkhaeuser, Boston 1991

[6] L.Bombelli, J.Lee, D.Meyer, R.Sorkin: Phys.Rev.Lett. 59(1987)521

A.P.Balachandran,G.Bimonte, E.Ercolesi, G.Landi, F.Lizzi, G.Sparano, P.Teotonio-Sobrinho: "Finite Quantum Physics...", Journ.Geom.Phys. 18(1996)163

[7] G.'t Hooft: “... Deterministic and Quantum Mechanical Systems”, J.Stat.Phys. 53(1988)323,

Nucl.Phys. B342(1990)471 
[8] T.Nowotny, M.Requardt: "Dimension Theory on Graphs and Networks", J.Phys.A:Math.Gen. 31(1998)2447, hep-th/9707082

[9] T.Nowotny, M.Requardt: "Pregeometric Concepts on Graphs and Cellular Networks", invited paper to appear J.Chaos, Solitons and Fractals, hep-th/9801199

[10] M.Requardt: "A New Approach to Functional Analysis on Graphs...", hepth/9708010

[11] M.Requardt: "Emergence of Space-time on the Planck Scale...", (preliminary version) hep-th/9610055

[12] C.Castro: "Beyond Strings...", hep-th/9707171

[13] L.Nottale: "Fractal Space Time and Microphysics...", World Scientific Publ., Singapore 1992

[14] A.Ashtekar: "Mathematical Problems of Non-Perturtbative Quantum General Relativity", Les Houches summer school on Gravitation and Quantization 1992 or gr-qc/9302024

[15] M.E.Agishtein, A.A.Migdal: "Simulation of 4-dimensional Simplicial Quantum Gravity as Dynamical Triangulation", Mod.Phys.Lett. A7(1992)1039

[16] R.M.Williams, P.A.Tuckey: "Regge Calculus...", Class.Quant.Grav. 9(1992)1409

[17] V.de Bakker: "Simplicial Quantum Gravity", Thesis, hep-lat/9508006

[18] T.D.Lee: "Discrete Mechanics", Int.School of Subnuclear Physics, Erice 1983

[19] B.Bollobás: "Graph Theory", Graduate Texts in Mathematics, Springer N.Y. 1979

[20] T.Toffoli, N.Margolus: "Cellular Automaton Machines", MIT Pr., Cambridge Mass. 1987

[21] F.Markopoulou, L.Smolin: "Causal Evolution of Spin Networks", Nucl.Phys. B508(1997)409 or gr-qc/9702025,

F.Markopoulou: "Dual Formulation of Spin Network Evolution", gr-qc/9704013

[22] R.Borissov: "Graphical Evolution of Spin Network States", Phys.Rev. D55(1997)6099 or gr-qc/9606013

[23] A.Connes: "Non-Commutative Geometry", Acad.Pr., N.Y. 1994

[24] J.Madore: "Non-Commutative Differential Geometry and its Physical Applications", Cambridge Univ.Pr., Cambridge 1995 
[25] R.Coqueraux: "Non Commutative Geometry and Theoretical Physics", Journ.Geom.Phys. 6(1989)425 and loc.cit. 11(1993)307

[26] A.Dimakis, F.Mueller-Hoissen: "Discrete Differential Calculus...", J.Math.Phys. 35(1994)6703; H.C. Baehr, A,Dimakis, F.MuellerHoissen: J.Phys.A:Math.Gen. 28(1995)3197

[27] M.Requardt: "Discrete Mathematics and Physics on the Planck-Scale...", hepth/9605103

[28] T.Nowotny: Diploma Thesis, Goettingen 1998

[29] E.H.Spanier: "Algebraic Topology", McGraw-Hill N.Y. 1966

[30] J.Stillwell: "Classical Topology and combinatorial Group Theory", Springer N.Y. 1993

[31] M.Dubois-Violette,J.Madore,T.Masson,J.Mourad: "On Curvature in Noncommutative Geometry", Journ.Math.Phys. 37(1996)4089

[32] G.A.Edgar: "Measure, Topology, and Fractal Geometry", Springer, N.Y. 1990, or

K.Kuratowski: "Topology" Vol.1, Acad.Pr., N.Y. 1966 